\title{
Chief Nurse in WHO-Führungsteam berufen
}

\section{Pflege wieder hochrangig vertreten}

» Im Rahmen der 68. Sitzung des WHO Regional-Komitees West-Pazifik in Brisbane/Australien, wurde Elizabeth Iro als Chief Nursing Officer bei der WHO ins Führungsteam von Generaldirektor Dr. Tedros Adhanom Ghebreyesus berufen. Dies löst auch in Deutschland große Zustimmung aus. So erklärte der DBfK: „Für diese Position haben die weltweiten Pflegeberufsverbände lange kämpfen müssen. Es ist längst überfällig, dass die Stimme und Perspektive der professionell Pflegenden im Stab des WHO-General- direktors gehört wird und mit entscheidet. Die zu lösenden Probleme aller Gesundheitssysteme sind bei weitem zu groß und zu komplex, als dass man auf diese Kompetenz verzichten könnte."

Frau Iro bringt als ,Registered Nurse“ und Gesundheitsministerin der CookInseln mehr als 30 Jahre Berufserfahrung im Bereich Public Health der gesamten Pazifik-Region mit. Als Ministerin hat sie wichtige Reformen durchgeführt, um das Gesundheitssystem ihres Landes zu stärken. Zudem war sie lange Präsidentin des Pflegeberufsverbands der Cook-Inseln, der auch Mitglied im Weltverband der Pflegeberufe ICN ist. Der WHO-Generaldirektor bekräftigte: „Pflegefachpersonen spielen eine entscheidende Rolle - nicht nur in der Gesundheitsversorgung von Millionen von Menschen in der ganzen Welt, sondern auch bei der Umsetzung von Gesundheitspolitik, der Gesundheitsförderung in Gemeinschaften und der Unterstützung von Patienten und Familien.“

www.who.int; www.dbfk.de

\section{Rund um die Uhr erreichbar: Medizinische Kinderschutzhotline}

\section{Kollegiales Beratungsangebot für Angehörige der Heilberufe}

") Das Gesundheitswesen spielt eine wichtige Rolle im System des institutionellen Kinderschutzes, besonders auch bei der Aufdeckung von Hilfebedarfen und Gefährdungslagen bei Säuglingen und Kleinkindern. Die Beratung von Fachkräften der Heilberufe rund um die Uhr durch eine ausreichende Anzahl zuverlässig erreichbarer Kinderschutzfachkräfte mit Erfahrungen im medizinischen Bereich, ist für einen funktionierenden Kinderschutz besonders relevant.

Die „Medizinische Kinderschutzhotline" ist ein kostenfreies kollegiales Beratungsangebot für Fachkräfte der Heilberufe. Sie ist sieben Tage die Woche/24 Stunden erreichbar bei Verdachtsfällen auf Kindeswohlgefährdung in medizinischen Not- und Akutsituationen in Kliniken und Praxen. Das Angebot soll dabei helfen, die un-

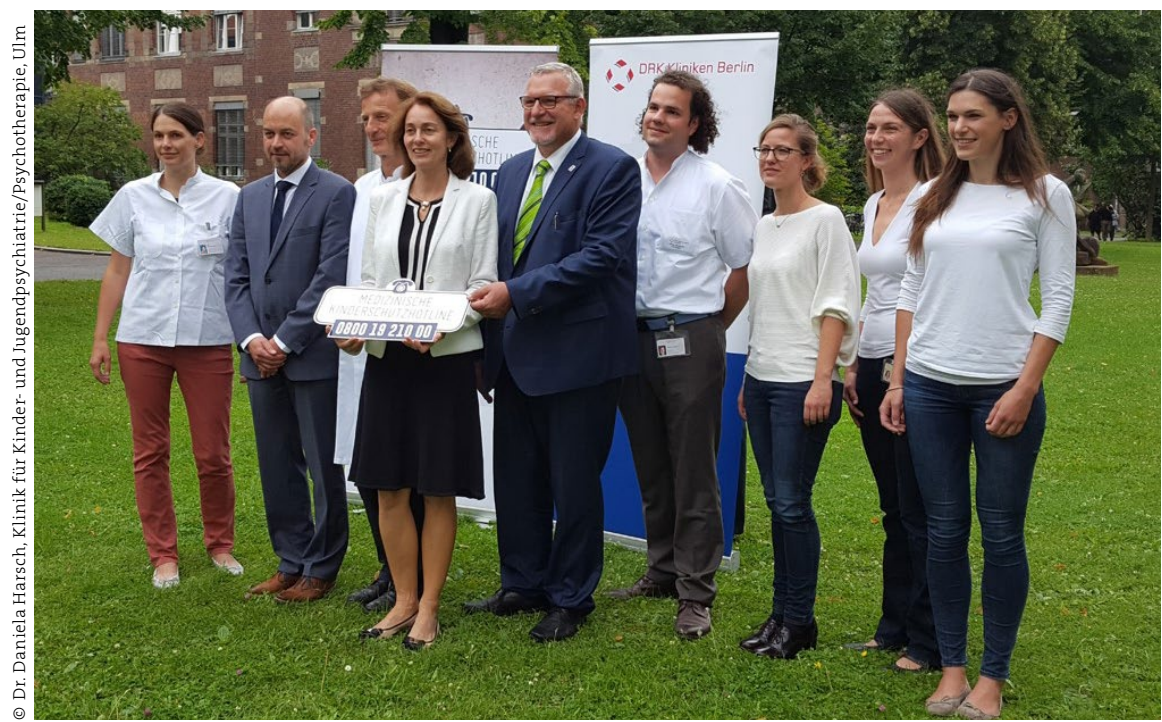

Hoher Besuch: Bundesfamilienministerin Dr. Katarina Barley beim Start der Medizinischen Kinderschutzhotline in den DRK Kliniken Berlin, rechts daneben Projektleiter Professor Jörg M. Fegert von der Klinik für Kinder- und Jugendpsychiatrie/Psychotherapie Ulm. terschiedlichen Fachsprachen und Herangehensweisen von Gesundheitswesen und Kinder- und Jugendhilfe zusammenzuführen. Verständigungsprobleme, die zu Lücken im Kinderschutz führen können, sollen so geschlossen werden. Die Beratung kann beispielsweise zum möglichen weiteren Vorgehen im konkreten Fall oder zur Gesprächsführung mit Eltern erfolgen. Die Fallverantwortung verbleibt stets bei den Anrufenden.

Die Beratungstätigkeit erfolgt durch Medizinerinnen und Mediziner mit einschlägigem Hintergrundwissen. Um einen hohen fachlichen Standard zu gewährleisten, ist ein mit einer Fachärztin oder einem Facharzt besetzter Hintergrunddienst eingerichtet. Neben einer begleitenden Forschung zur Qualitätssicherung erfolgt am Ende der Projektlaufzeit auch eine externe Evaluation des Projektes.

Das vom Bundesministerium für Familie, Senioren, Frauen und Jugend (BMFSFJ) geförderte Projekt hat eine Laufzeit von drei Jahren. Projektleiter ist Prof. Dr. Jörg M. Fegert von der Klinik für Kinder- und Jugendpsychiatrie/Psychotherapie des Universitätsklinikums Ulm. Die Hotline ist seit Juli 2017 bundesweit verfügbar. Alle Informationen zum Projekt online unter

www.kinderschutzhotline.de 